\title{
Acceleration of tissue maturation by mechanotransduction-based bioprinting
}

\author{
Ashkan Shafiee $\odot,{ }^{1, *}$ Jareer Kassis $\odot,{ }^{1}$ Anthony Atala, ${ }^{1}$ and Elham Ghadiri ${ }^{1,2,3}$ \\ ${ }^{1}$ Wake Forest Institute for Regenerative Medicine, Wake Forest School of Medicine, North Carolina 27101, USA \\ ${ }^{2}$ Department of Chemistry, Wake Forest University, North Carolina 27109, USA \\ ${ }^{3}$ Comprehensive Cancer Center, Wake Forest School of Medicine, North Carolina 27101, USA
}

(Received 9 July 2020; revised 18 October 2020; accepted 17 November 2020; published 5 January 2021)

\begin{abstract}
The growing number of patients who require organ transplants, combined with the low number of organ donors, has resulted in organ shortages; therefore, the fabrication of human tissues and organs is an urgent need. However, the time required to fabricate an organ may result in risky delays for end-stage patients who urgently require transplants. During bioprinting, the maturation of the engineered tissue that is required before it is ready for implantation is lengthy. Here we use a previously introduced microscopic and mathematical model, the "zipper CAMs" (for cell adhesion molecules), to investigate the effective parameters involved in tissue dynamics. In our current study, we validated the ability of our model to accelerate the tissue maturation process. Our model shows that exploiting cellular mechanotransduction can accelerate post-printing tissue maturation. To verify this prediction experimentally, we devised a mechanotransduction-based bioprinter that accelerates the production of tissues by speeding up the fusion bioink particles. The mathematical microscopic model and the bioprinter described herein are expected to be highly useful in cell biology, tissue engineering, and biofabrication.
\end{abstract}

DOI: 10.1103/PhysRevResearch.3.013008

\section{INTRODUCTION}

Organ shortage is a critical healthcare problem given an aging population as well as higher rates of diseases and injuries [1-3]. Meanwhile, the low number of organ donors has lengthened the wait time for organ transplantations [4]. Over the past 20 years, several innovations and clinical trials have produced tissue and organ engineering methods in pursuit of biofabricating human organs. Successful applications of tissue engineering have been reported in the fabrication and transplantation of animal and human organs [5-7]. Biological constructs can typically be built from a patient's own cells (autografts), or those of genetically nonidentical individuals (allografts). Currently, immunosuppressive drugs are used to prevent transplant rejection; however, approximately $20 \%$ of patients on transplantation lists are those who have already received organs from healthy or deceased human donors but require new organs after rejection $[8,9]$. To that end, autologous tissue engineering would remedy both the organ donor shortage and chronic rejection of transplanted organs [10]. Nevertheless, given that organ engineering may involve cell biopsy, proliferation, tissue preparation, biofabrication, maturation, and implantation, the long duration of the procedure can place the lives of patients in urgent need of new organs

\footnotetext{
*Author to whom correspondence should be addressed: ashafiee@wakehealth.edu

Published by the American Physical Society under the terms of the Creative Commons Attribution 4.0 International license. Further distribution of this work must maintain attribution to the author(s) and the published article's title, journal citation, and DOI.
}

at risk. Therefore, accelerating each step of the fabrication sequence is desirable. This, however, requires a deep understanding of the underlying numerous biological and physical processes [11].

Among all biofabrication techniques currently available, bioprinting holds promise owing to its unique advantages, such as reproducibility, computer-aided fabrication, speed, and accuracy of material dispensing [12,13]. For example, scaffold-free bioprinting can produce biological structures such as blood vessels and nerves, wherein small pieces of spherical or cylindrical tissues (cellular bioinks) are delivered in appropriate geometries and designs [14-16]. After bioprinting is complete, cellular self-assembly (tissue fusion) produces mature biological constructs that are transplantable [17-19]. For cellular self-assembly, tissue engineers recreate near-physiological conditions to drive cellular interactions toward the final product [20]. However, most bioprinting processes, including the fabrication of blood vessels and nerves, have involved simple designs that eliminate external forces; this resulted in studying cellular self-assembly in the absence of stimulating forces $[14,15,18]$. Eliminating such external forces rendered this approach nonrepresentative of actual cellular conditions, as it has been shown that natural cellular mechanotransduction is used to regulate numerous biological processes ranging from cell proliferation and differentiation to embryogenesis [21]. Physical forces and mechanics are among the most important factors in developmental and cellular biology [22,23]; for example, flow shear induces changes in both the cytoskeletal structure and gene expression patterns of cultured cardiac endothelial cells [24]. Mechanotransmission occurs throughout the mechanosensitive adhesion process and elicits cadherin- and integrin-mediated mechanotransduction by transmitting forces between cells and/or the 


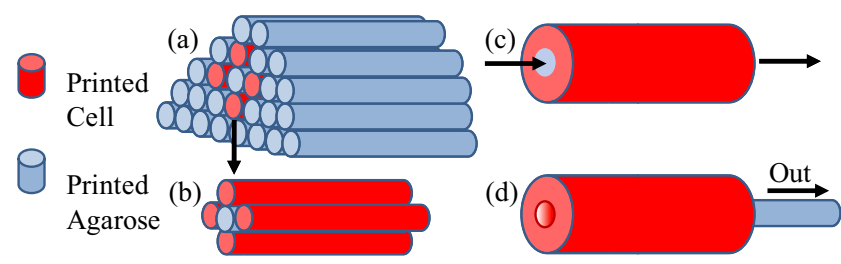

FIG. 1. Schematic view of printing tubular structures such as blood vessels. (a) Tubular structures can be obtained by printing agarose (blue cylinders) and cylindrical cellular bioinks (red cylinders) layer by layer. (b) A closer look at the center of the printed constructs with cellular bioinks and agarose inside. (c) The continuous tissue construct forms through cellular self-assembly and the fusion of the discrete bioink particles. (d) By removing the agarose rod to which cells do not adhere, a tubular cellular structure is achieved. The inner diameter of the lumen can be controlled by the numbers and diameters of the printed cylindrical bioinks and agarose rods [14].

cell and the extracellular matrix [25]. Consequently, forceinduced functions (such as enzymatic reactions and protein conformational changes) occur, leading to mechanosignaling. It has also been observed that cellular mechanotransduction contributes to cardiomyocyte formation and functionality [26]. Moreover, cell functions can be altered by an external force; the differentiation of stem cells into either neurons or osteoblasts can be fine-tuned using different cell culturing substrate stiffnesses [27].

Given that tissues demonstrate liquidlike behaviors $[11,28,29]$, we applied tissue-liquidity notion to investigate cellular self-assembly. During bioprinting, the cellular bioinks (minitissues in our case) undergo such self-assembly, including tissue fusion, akin to that of liquid drops. Hence, we regarded the viscoelastic properties of tissues as analogous to liquids when considering the former's characteristic fusion times; such fusion is the most important cellular self-assembly process. Over the course of the fusion process, cellular bioinks rearrange and develop essential connections as well as an extracellular matrix to cement the integrity of the structure and become a sturdy biological construct. This process is schematically shown in Fig. 1.

It has been demonstrated that the fusion between two cellular bioinks can be quantified in terms of the characteristic fusion time, which in turn allows the determination of the optimum time for tissue maturation $[14,17,19]$. Therefore, tissue engineers can compare tissue fusion as a means to investigate tissue maturation quantitatively. A shorter-than-optimal maturation time may result in weakened mechanical properties, possibly leading to the destruction of the bioprinted structure under physiological conditions (e.g., low burst pressure of a tubular construct). On the other hand, too long a delay may result in a necrotic core within the bioprinted structure. As such, the characteristic fusion time provides a quantitative gauge to investigate the fusion process after bioprinting, and has been used by several researchers [28-30]; the shorter the characteristic fusion time, the faster the tissue maturation.

Here, we describe the use of mechanobiology to accelerate the maturation of bioprinted tissues. To assess the cellular level interactions during the tissue maturation process, we investigated the kinetics of cell adhesion molecules as established by Bell [31], whose model provides a mathematical means to predict the rate of bond formation between two cells. Furthermore, to investigate the dynamics of multicellular systems, we employed a previously developed microscopic and mathematical model (the zipper model) with which we calculated the force and energy required for self-assembly during tissue maturation [32,33]. We used statistical mechanics to investigate the behavior of printed bioinks undergoing cellular self-assembly. A three-dimensional (3D) zipper consisting of several links was employed to describe the fusion process; each closed link in the zipper represented a bond between the adhesion molecules on the membranes of cells present in each of the two fusing bioinks. This analogy facilitates the investigation of cellular self-assembly on both multicellular and single-cell scales.

In this work, we used the rate of bond formation during the biased attachment of two single cells. Moreover, we generalized the zipper model for multicellular systems when exposed to an external force. Our mathematical model predicted that the characteristic fusion time of cellular bioinks decreases owing to external forces. Therefore, we devised a special-purpose bioprinter with which tissue maturation was accelerated via an external force. The characteristic fusion times of the bioprinted cellular bioinks were measured and compared for two different experimental configurations: in the presence and absence of an external force. As predicted by our model, the applied external force led to a more than threefold accelerated maturation process. These empirical results validate our microscopic and mathematical model, which can therefore be used to predict and control tissue formation. To date, bioprinters have not been installed in operating rooms nor have they demonstrated clinical applicability. Understanding the underlying physics of bioprinting technology may help transition the field from a "trial and error" stage to a "predict and control" phase, and accelerate the introduction of bioprinters into clinical settings.

\section{MATERIALS AND METHODS}

\section{A. Cell culture}

We used human skin fibroblasts (HSFs) that were purchased from the American Type Culture Collection (CRL2522; ATCC, Manassas, VA). Cells were cultured in Dulbecco's Modified Eagle Medium with Ham's F12 Nutrient Mixture, antibiotics (penicillin/streptomycin and gentamicin), $2 \mathrm{mM}$ glutamine, $0.1 \mathrm{M}$ sodium pyruvate (all chemicals were purchased from Life Technologies Gibco; Green Island, NY) containing 20\% fetal bovine serum (FBS; Thermo Fisher Scientific, Waltham, MA) on $0.5 \%$ gelatin-coated dishes (Sigma, St. Louis, MO) and were maintained at $37^{\circ} \mathrm{C}$ in a humidified atmosphere containing $5 \% \mathrm{CO}_{2}$. In each subculture, the cells were washed twice with phosphate-buffered saline and detached using a $0.1 \%$ trypsin-EDTA solution (Life Technologies Gibco; Green Island, NY and Sigma, St. Louis, MO, respectively). Cylinders were fixed in $4 \%$ paraformaldehyde (Alfa Aesar, Ward Hill, MA). To ensure that all cells were at the same passage number, cultures, at a particular passage, were cryopreserved upon reaching a sufficient number 
(a)

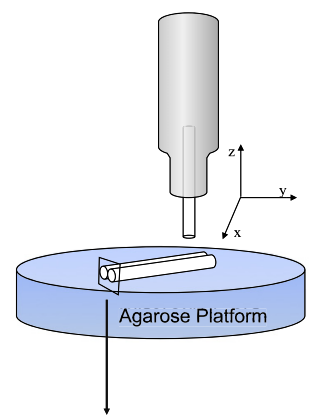

(b)

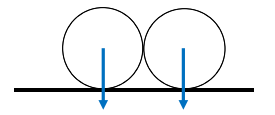

(c)

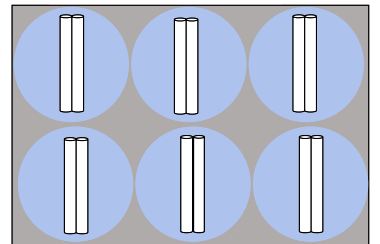

(d)

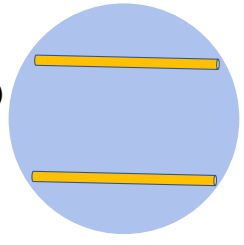

(e)

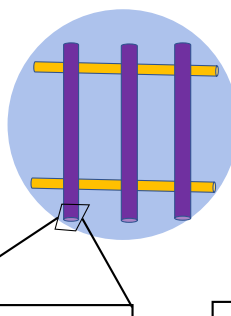

(f)

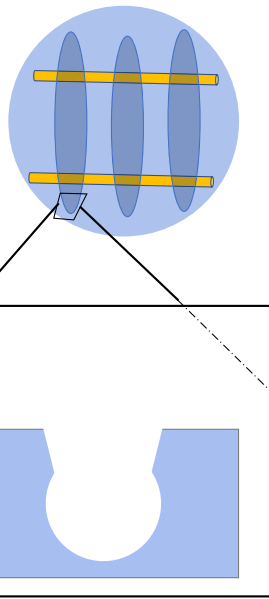

(g)

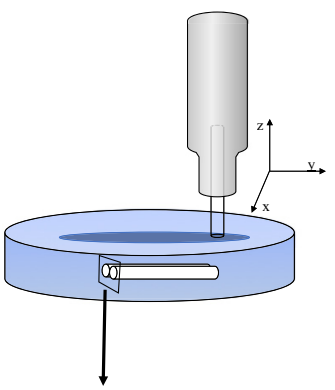

(h)

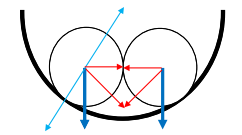

(i)

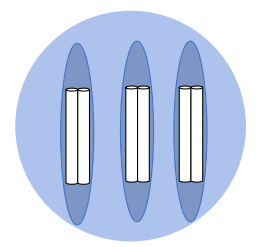

Cross section: Removing the glass tube

FIG. 2. Bioprinting in the absence and presence of an external force: (a) Cellular bioinks were extruded according to the normal bioprinting protocol using flat surfaces to investigate the fusion without external forces. (b) Side view of two fusing cellular bioinks on a flat surface of agarose. (c) Six well dishes (3.5 cm diameter of each well) were used and covered by agarose to create flat surfaces. Cellular bioinks were printed in pairs in each well, and the fusion process was followed at different time points. (d)-(f) In mechanotransduction-based bioprinting, grooves are used to apply external forces on fusing cellular bioinks. Creating the glass foundation in 10-cm Petri dishes using capillary micropipettes (yellow cylinders). Glass tubes with 1.35-mm outer diameters were placed on the glass foundation, and the configuration was poured with liquid agarose. The grooves were created by removing the $1.35-\mathrm{mm}$ outer diameter glass tubes and two cylinders were printed in each groove. (g) Bioprinting cellular bioinks inside grooves. (h) Side view of two fusing cellular bioinks. Printed cellular bioinks experience an external force. (i) Top view of printed cellular bioinks in the presence of grooves.

of cells. After trypsinization, cells were pelleted via $5 \mathrm{~min}$ of centrifugation at 2,000 rpm and resuspended in a freezing medium consisting of $10 \%$ dimethyl sulfoxide (Thermo Fisher Scientific) and 90\% FBS. The solution was transferred to cryogenic screw cap vials (Thermo Fischer Scientific) and placed in Nalgene freezing canisters (Thermo Fischer Scientific) containing isopropyl alcohol; and incubated in a $-80^{\circ} \mathrm{C}$ freezer for $24 \mathrm{~h}$. Subsequently, cells were stored in a liquid nitrogen tank until use for cellular bioink preparation.

\section{B. Fabrication of cellular bioinks}

Multicellular aggregates or cellular bioinks were fabricated using HSFs in cylindrical cultures using the cylinder fabrication method. HSFs have previously been validated for use in fabrication and transplantation of autologous and allogenic human tissue-engineered vascular grafts [34,35]. The structures were successfully used for patients who were receiving hemodialysis via access graft. The autologous blood vessels showed a mean burst pressure of $3512 \mathrm{~mm} \mathrm{Hg}$; however, their fabrication required a mean of 7.5 months. To fabricate bioprinted tubular structures, cylindrical bioinks can be printed layer by layer with agarose rods in a tubular geometry as shown in Fig. 1. After the cellular self-assembly and fusion are complete, the bioprinted tubular construct is ready for the next steps, such as in a bioreactor for functionality training and eventually implantation. Therefore, to mimic the fusion of cylindrical bioinks, we designed two bioprinting protocols; normal and mechanotransduction-based as described below.

In this work, cells were trypsinized and centrifuged in $15 \mathrm{~mL}$ tubes for $5 \mathrm{~min}$ at $2000 \mathrm{rpm}$. Subsequently, the medium was discarded, and the resulting cell pellet was gently resuspended in $300 \mu \mathrm{L}$ of fresh medium and transferred to a $1 \mathrm{~mL}$ syringe. The syringe was capped with a rubber stopper and was centrifuged for $2 \mathrm{~min}$ at $2000 \mathrm{rpm}$. The supernatant forming in the syringe was siphoned off via a hole made by a needle in the syringe at the pellet-supernatant interface. The remaining cell paste was then aspirated into capillary micropipettes with inner diameters of $500 \mu \mathrm{m}$ and incubated for $15 \mathrm{~min}$. As-prepared cylindrical cellular bioinks were bioprinted with a constant pressure that dispensed material at $5 \mathrm{~mm} / \mathrm{s}$ either on agarose flat surfaces or grooves (Fig. 2).

\section{Fusion of cylindrical HSF bioinks}

Fusion experiments with cylinders were performed using two different methods: cylinders were printed either next to each other on flat agarose surfaces or contiguously into grooves (with semicircular cross sections) in an agarose platform (for details see Fig. 2). The fusion processes were recorded at different time points to obtain characteristic fusion times. To record the fusion process in both cases, the medium was removed and samples were fixed under a chemical hood 
(a)

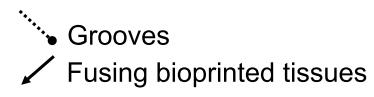

(b)

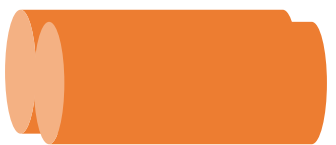

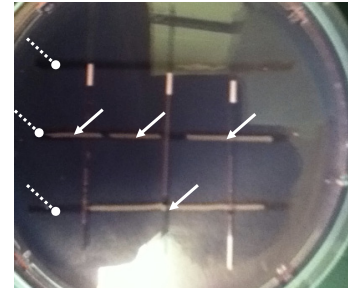

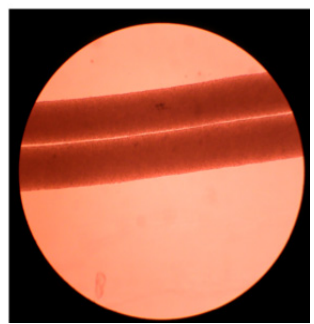

(c)

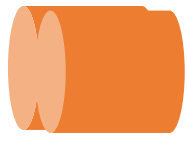

(d)
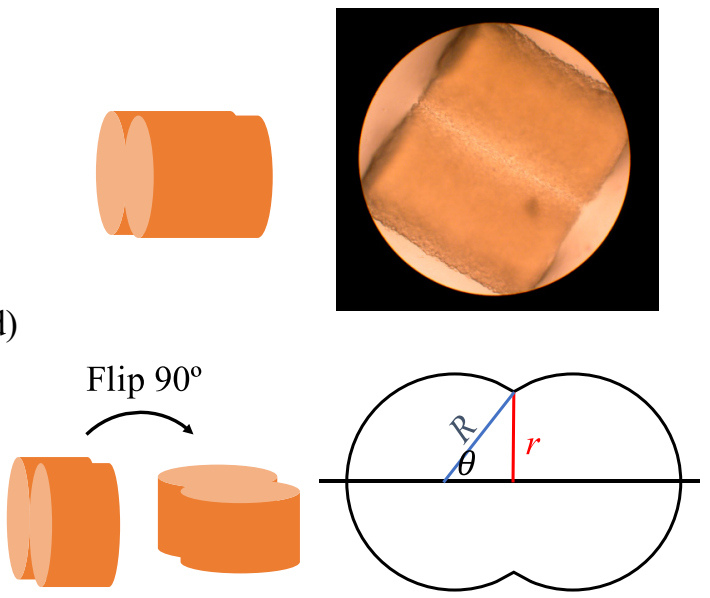

FIG. 3. (a) Pairs of cylindrical bioinks of human skin fibroblast are printed in previously prepared grooves. (b) Schematics of two fusing cellular bioinks as well as a top view of a fusing pair cylindrical bioinks (at $t=0$ ) under a microscope. (c) Schematics of small pieces of fused bioinks as well as a top view of fused bioinks under a microscope. (d) Fused cylindrical bioinks are cut into small pieces using surgical scalpels and flipped $90^{\circ}$ in order to image the cross section of the fusion under a microscope attached to a camera. Essential parameters to investigate the fusion are introduced as $R, r$, and $\boldsymbol{\theta}$. The fusion process will be studied quantitatively by plotting the $\sin \boldsymbol{\theta}$ vs time.

using $4 \%$ paraformaldehyde for 30 min, followed by three rinses with phosphate-buffered saline for $20 \mathrm{~min}$ each. After fixation, fused pairs of cylinders were placed on $10-\mathrm{cm}$ Petri dishes covered with $15 \mathrm{~mL}$ solid agarose and were embedded in $15 \mathrm{~mL}$ of soft agarose gel. The dishes were placed in a refrigerator until imaging.

For imaging, the fused cylinders were cut into small pieces using surgical scalpels. The pieces were flipped at $90^{\circ}$ to align their cross sections with the flat surface (Fig. 3). The contours of the fused cross sections were photographed with a digital camera (Nikon, Japan) attached to an Olympus IX70 (Center Valley, PA) inverted microscope.

For imaging fused cylindrical bioinks, the samples were sacrificed and fixed for each time point. Thus, a large number of pairs were prepared at $t=0$ and were kept in an incubator to fuse. Consequently, at each time point, several samples were fixed and prepared for imaging, and the remaining samples were retained for other time points. This process was continued until images were prepared at all the desired time points.

\section{RESULTS AND DISCUSSION}

The fusion process for cylinders was performed using two different protocols. The characteristic fusion time $(\tau)$ for both protocols were obtained using images of fusion experiments at different time points that were recorded under a microscope. The images then were processed to identify essential parameters [presented in Fig. 3(d)], including $r$ and $\theta$. The characteristic fusion time could be obtained by plotting $\sin \theta$ versus time [18]. Images of several samples were analyzed at each time point, with the average represented as $\sin \theta$ per time point.
Flat surface. To extract the characteristic fusion time due exclusively to the coalescence of the two cylinders as driven by their apparent liquid properties, the fusion experiments were performed on a flat surface. Figure 4(a) illustrates the time evolution of the shape of the cylinders' cross section in one particular sequence. The $\sin \theta$ vs time was plotted by averaging the results over six samples (i.e., images) at each time point [Fig. 4(b)]. Collecting data at $2 \mathrm{~h}$ after the initiation of fusion could not be performed consistently. Even if some samples could be photographed, it would be challenging to handle, fix, and image them at $2 \mathrm{~h}$, as bioinks in these samples showed poor fusion due to the loose connection between them at this early time point. Given this rationale, we ignored the datapoint for $2 \mathrm{~h}$ and plotted the $\sin \theta$ vs time as shown in Fig. 4(b). The exponential fit $(\sin \theta=1-\exp (-t / \tau))$ of these data resulted in a characteristic fusion time equal to $9.76 \mathrm{~h}$ (standard deviation [SD]: 1.027), and $t_{0}$ was 5.23 $\mathrm{h}$, which is a fitting parameter for the exponential fit and provides the de facto time when fusion has commenced. Experimentally, the fusing bioinks are easy to separate at early time points but are impossible to dissociate without a destructive force (such as scalpel) after attachment has commenced.

Fusion in grooves. Figure 5 shows $\sin \theta$ vs time for the fusing experiment inside grooves. The obtained characteristic fusion time $\tau$ for this protocol was 3.05 hours (SD: 0.65 hours), and $t_{0}$ was $1.12 \mathrm{~h}$.

As can be observed when comparing Figs. 4 and 5, the fusion process reached $70 \%$ of its maximum in $4 \mathrm{~h}$ when the bioinks were fusing inside grooves. The discrepancies between characteristic fusion times for bioinks printed on flat surfaces and grooves are also notable when comparing the quantitative values of 9.76 and $3.05 \mathrm{~h}$. 


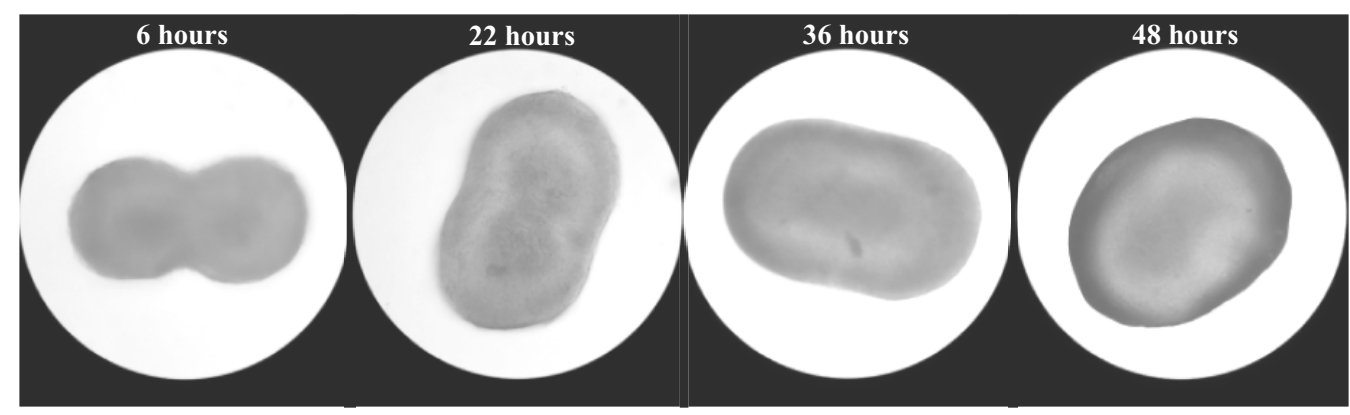

(a)

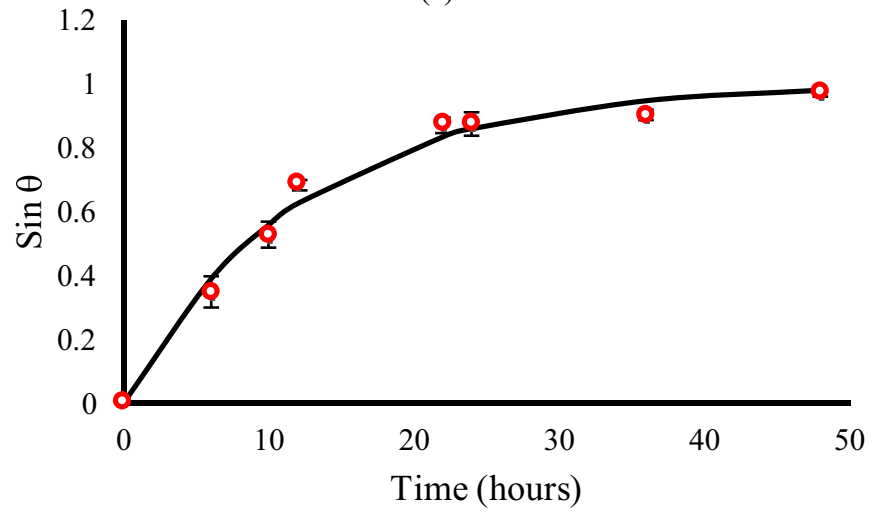

(b)

FIG. 4. (a) Cross-sectional images of fusing cylinders at selected time points. The images are acquired by cutting small sections of fusing cylinders and flipping them by $90^{\circ}$ to image the cross section of the fusion area as depicted in Fig. 3(d). (b) sin $\boldsymbol{\theta}$ vs time for cylindrical human skin fibroblast bioinks printed on flat agarose surface. Red circles represent the average of $\sin \theta$ over six samples at each time point. The solid line is the theoretical fit of the data. The minimized residual sum of squares was 0.0098 .

Considering all aspects of these two experiments, including the same cell type, medium, and image processing, this difference in the characteristic fusion times stems from the external forces exerted by the agarose wall on cellular bioinks. The fusing aggregates slightly press against each other owing to different external forces, such as rotational torque and gravity (note that the bottom of the groove is not flat). Thus,

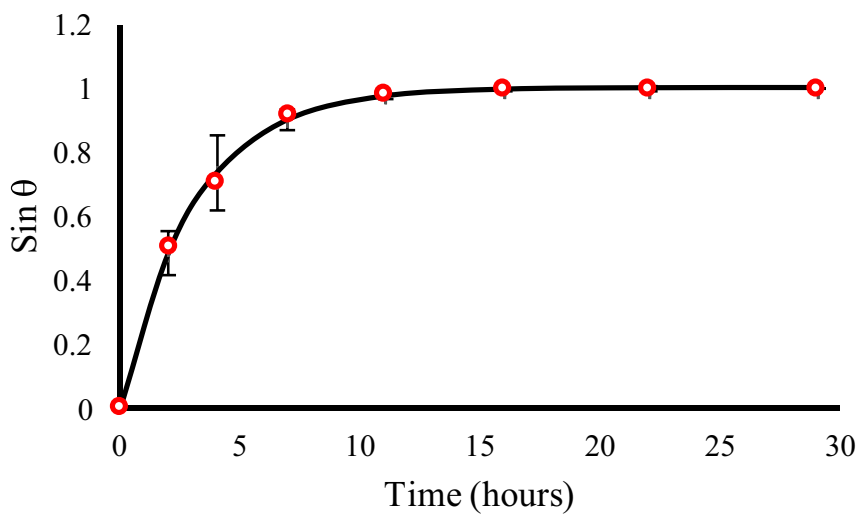

FIG. 5. $\sin \theta$ vs time for cylindrical human skin fibroblast bioinks printed onto grooves. Red circles represent the average of $\sin \theta$ over five samples at each time point. The solid line is the theoretical fit curve. The minimized residual sum of squares was 0.0014 . the characteristic fusion time found in the process in the groove reflects not only the genuine coalescence of the two cylinders but also the distortion of the fusion contour due to an additional mechanical force (compared to the process on flat surface), which in turn may elicit a cascade of signaling processes through intracellular mechanotransduction.

It was shown that the higher the apparent tissue surface tension (ATST) of the bioink, the faster the fusion [33]. This is due to a theoretically established relationship according to which the ATST can be expressed as $c J N$, where $J$ is the effective characteristic binding energy between cell adhesion molecules, $N$ is the number of these contacts (per unit area), and $c$ is a dimensionless constant [28]. Moreover, it has been demonstrated experimentally that the ATST of cellular aggregates composed of the same cell type varies linearly with the number of cell adhesion molecules present on the membranes of the composing cells: the greater the number of cell adhesion molecules, the higher the ATST [36]. Therefore, cell adhesion molecules play an important role in tissue fusion and formation.

We previously established a microscopic model for the fusion of the minitissue multicellular aggregates based on the adhesion molecules of the composing cells [32,33]. In brief, our model states that the fusion process consists of a cascade of cellular attachments. The adhesion molecules of cells in juxtaposed cylinders break some bonds with cells on the same cylindrical bioink and develop new bonds with cells 
(a)
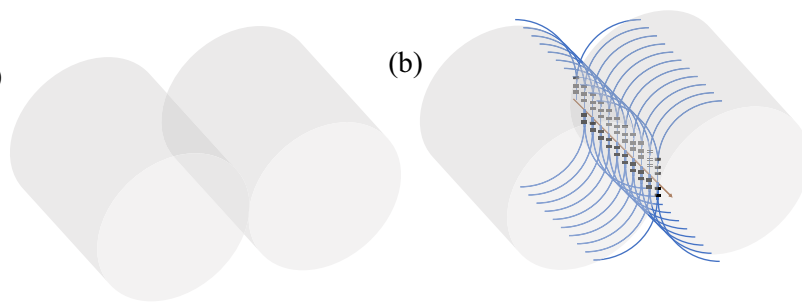

(c)

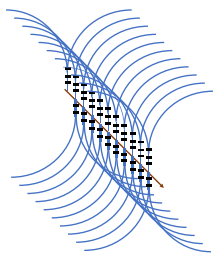

(d)

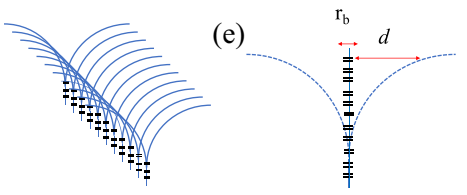

(f)
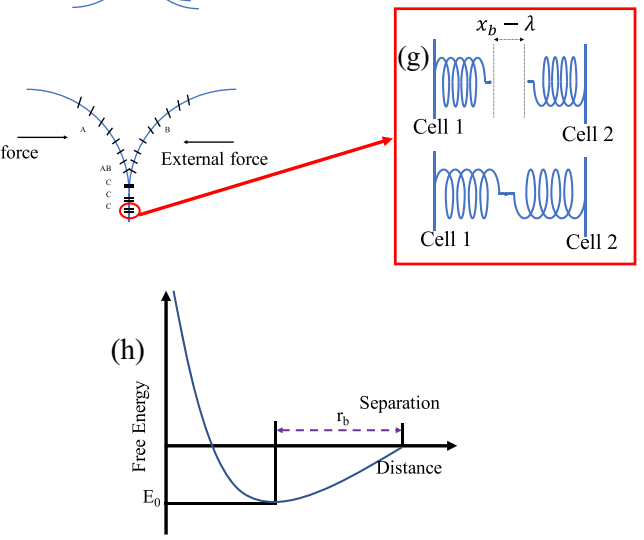

FIG. 6. The zipper model to investigate the rate of fusion. (a)-(c) The cascade of cellular attachments on the surface of two fusing cellular cylindrical bioinks can be considered as two series of closing zippers, one above and another one below the contact line. Analysis of the model in terms of statistical mechanics [32] provides the force and energy of fusion. (d) A series of zippers above the contact line. (e) Each zipper represents the attachment of consecutive cells on apposing cylindrical bioinks. The dashed curves represent the original locations of the adhesion molecules on the bioinks' surfaces. The solid vertical line depicts the final closed zipper when the fusion of bioinks is complete. (f) Cell adhesion molecules on apposing bioinks can develop a bond or link. If the pair of adhesion molecules are far, the link is in configuration 1 with energy zero, whereas if they are sufficiently close [around $r_{b}$ in panel (h)], the link is in configuration 2 with energy $\varepsilon$. An external force may provide a biased cellular attachment using mechanotransduction for fusion. Red square: A closer look at the red circle in (f). Adhesion molecules can be considered springs with a deviation bond length; $\left|x_{b}-\lambda\right|$. (h) Molecules must be within an appropriate distance $\left(r_{b}\right)$ to be able to form a closed link.

on the opposing cylindrical bioink. After completing these cellular attachments, new cells now come into the vicinity of each other and the above process repeats; this cascade of cell attachment continues until fusion is complete (Fig. 6).

The post-bioprinting maturation process can be investigated at both the multicellular and cellular scales. In the macroscopic view (dynamics of multicellular system and cellular attachments), the cascade of cell attachments can be considered a closing zipper (Fig. 6). The teeth (i.e., cells) on both sides engage each other (i.e., form a link) to form a chain and consequently close the zipper. Understanding this analogy may simplify the complicated process of multicellular self-assembly and facilitate mathematical analysis. The model allows the calculation of the energy of self-assembly by summing the energy of each individual zipper link element. Each link has two possible configurations: (1) open with energy zero (ground state) or (2) closed with energy $\varepsilon$. The zipper models fusion in a plane; consequently, 3D fusion can be described as a set of zippers (Fig. 6), resulting in the total energy of the system (for further details see Ref. [32]):

$$
\begin{aligned}
\langle E\rangle_{\text {total for cyl }} & =\frac{2 \ell}{\kappa}\langle E\rangle \\
& =\frac{-2 \ell}{\kappa}\left(\frac{\varepsilon(N+1) e^{-(N+1) \beta \varepsilon}}{1-e^{-(N+1) \beta \varepsilon}}+\frac{\varepsilon e^{-\beta \varepsilon}}{1-e^{-\beta \varepsilon}}\right),
\end{aligned}
$$

where $\beta$ is the thermodynamic beta $\left(\beta=\frac{1}{k_{B} T}\right)$.

Here, we generalize the mathematical investigation of the cellular self-assembly to a more realistic situation in which there is an external force. The work of moving cells to the vicinity of counterpart cells in the opposite bioink is equal to the energy produced in chemical reactions $\left(\langle E\rangle_{\text {total }}\right)$. Therefore,

$$
\begin{aligned}
F & =N N_{b} f, \\
F d & =\langle E\rangle_{\text {total }}=N N_{b} f d, \\
f & =\left(\langle E\rangle_{\text {total }} / N N_{b} d\right) \\
& =\frac{-2 \ell}{\kappa N N_{b} d}\left(\frac{\varepsilon(N+1) e^{-(N+1) \beta \varepsilon}}{1-e^{-(N+1) \beta \varepsilon}}+\frac{\varepsilon e^{-\beta \varepsilon}}{1-e^{-\beta \varepsilon}}\right) .
\end{aligned}
$$

In Eq. (3), $F$ and $d$ represent the force and the cell displacement done by the work of self-assembly, respectively. These are the macroscopic quantities that are based on some microscopic quantities, including $f$ and $r$ that are the force required to rupture/develop a bond and the distance between molecules, respectively [Fig. 6(e)]. It is worth noting that the total force $F$ is the summation of $f$ for $N$ number of cells and $N_{b}$ number of bonded adhesion molecules on two attached cells, and $d$ is the distance that cells must relocate in order to be in the vicinity of other cells on the other bioink and start developing bonds.

In the microscopic (cellular level) view based on the dynamics of cell adhesion molecules, the rate of bond formation between cells can be estimated using Bell's model for specific cell-cell adhesion [31], according to which the predicted rate of bond formation is $\mathrm{A}+\mathrm{B} \underset{k_{-} k_{+}}{\leftrightarrow} \mathrm{C}$, where $k_{-}$and $k_{+}$are reverse and forward rate constants, respectively. In this case, Bell stated that the rate of bond formation can be found from

$$
\frac{d N_{b}}{d t}=k_{+}\left(N_{1}-N_{b}\right)\left(N_{2}-N_{b}\right)-k_{-} N_{b},
$$

where $N_{1}$ and $N_{2}$ are the number of available adhesion molecules on cell 1 and cell 2 , respectively. Here, we are investigating the adhesion of bioinks that consist of the same cell type (human skin fibroblast); therefore, we may consider that $N_{1}=N_{2}=Y$ :

$$
\frac{d N_{b}}{d t}=k_{+}\left(Y-N_{b}\right)^{2}-k_{-} N_{b} .
$$


Bell solved the rate constants for dissociating the bonds and separating two cells from each other. Numerous experimental studies observed the effect of external forces on cell separation; moreover, a few teams derived the mathematical equations for compressed cells and their attachment [37]. Nevertheless, the experimental work on the impact of external forces on cell attachment has not been a research focus [38], although tissue engineering and accelerating tissue maturation may be the most important applications of cell attachment under compression that have yet to be explored. Here, we consider adhesion of two single cells using an external force and generalize it for biased multicell attachment in multicellular systems, namely bioinks.

To compare the rate of bond formation for both printing protocols, one needs to calculate the reverse and forward rate constants $\left(k_{ \pm}\right)$with and without an external force. To that end, we may use the kinetic theory of the strength of solids to calculate the time required to rupture a bond:

$$
\left.\tau^{\prime}=\tau_{0} \exp \left[\left(E_{0}-\alpha f\right) / K_{B} T\right)\right] .
$$

In Eq. (7), $\tau_{0}$ is the reciprocal of the natural frequency of oscillation of atoms in a solid, $\alpha$ is an empirical value that shows the solid imperfection and structure (in units of length), $E_{0}$ is the energy of each bond (which is $\varepsilon$ in this case), and $f$ is the applied force per bond [Eq. (4)]. Many researchers have employed this relationship to obtain the lifetime of a bond in different cells [31]. It is worth noting that the reverse rate constant is the inverse of the lifetime for the situation in which the external force is zero.

$$
\left.\tau_{(f=0)}^{\prime}=k_{-}^{-1} \rightarrow k_{-(f=0)}=k_{-}^{0}=\frac{1}{\tau_{0}}\left[\exp (\varepsilon) / K_{B} T\right)\right]^{-1} \text {. }
$$

Furthermore, when there is an external force to dissociate two cells from each other the reverse rate constant becomes

$$
k_{-}(f)=k_{-}^{0} \exp \left[\alpha f / k_{B} T\right] .
$$

Here, the aim is to apply a force to facilitate cell attachment. Therefore, the forward rate constant is the important factor for this situation. Using the reverse rate constant and Boltzmann distribution, the forward rate constant can be obtained [37]. In statistical mechanics, a Boltzmann distribution is a probability distribution that provides the likelihood of a system being in a specific state as a function of the temperature of the system and the energy of that particular state. The Boltzmann distribution thus determines the probability that a system is in a particular state. This probability is based on that state's energy and the temperature of the system;

$$
\frac{p_{i}}{p_{j}}=e^{\frac{\varepsilon_{j}-\varepsilon_{i}}{k T}} .
$$

In Eq. (10), $p_{i, j}$ and $\varepsilon_{i, j}$ represent the probabilities and energies of states $i$ and $j$, respectively. Here, the energy for open states is zero and for closed states is $\varepsilon$,

$$
\frac{k_{+}}{k_{-}}=\frac{k_{+}^{0}}{k_{-}^{0}} \exp \left[\frac{0-\varepsilon}{k_{B} T}\right] .
$$

It was shown that cell bridges could be considered springs [37]. Therefore, using classical mechanics, the energy of a spring is $E=\frac{1}{2} \sigma x^{2}$, where $\sigma$ is the Hookean spring constant. Consequently, the energy of each bond or cell bridge can be considered $\varepsilon=\frac{1}{2} \sigma|x-\lambda|^{2}$, where $|x-\lambda|$ is the deviation bond length [Fig. 6(f)]. By using Eq. (10), the forward rate constant can be calculated as

$$
\frac{k_{+}}{k_{-}}=\frac{k_{+}^{0}}{k_{-}^{0}} \exp \left[\frac{-\sigma\left|x_{b}-\lambda\right|^{2}}{2 k_{b} T}\right],
$$

therefore, by using Eq. (9),

$$
\begin{aligned}
k_{+} & =\frac{k_{-} k_{+}^{0}}{k_{-}^{0}} \exp \left[\frac{-\sigma\left|x_{b}-\lambda\right|^{2}}{2 k_{b} T}\right] \\
& =k_{+}^{0} \exp \left[\frac{\alpha f}{k_{B} T}\right] \exp \left[\frac{-\sigma\left|x_{b}-\lambda\right|^{2}}{2 k_{b} T}\right] \\
& =k_{+}^{0} \exp \left[\frac{\sigma\left|x_{b}-\lambda\right|\left(\alpha-\frac{1}{2}\left|x_{b}-\lambda\right|\right)}{k_{B} T}\right] .
\end{aligned}
$$

We considered only two different scenarios: the presence or absence of an external force. Therefore, using Eq. (13), one discovers that the forward rate constant for cell attachment in the mechanotransduction-based bioprinting is higher than that with standard bioprinting protocol. By incorporating Eq. (13) into Eq. (6), we arrive at

$$
\begin{aligned}
\frac{d N_{b}}{d t}= & k_{+}^{0} \exp \left[\frac{\sigma\left|x_{b}-\lambda\right|\left(\alpha-\frac{1}{2}\left|x_{b}-\lambda\right|\right)}{k_{B} T}\right] \\
& \times\left(Y-N_{b}\right)^{2}-k_{-}^{0} N_{b} .
\end{aligned}
$$

Here, we deal with external force in the positive direction (to help the forward rate constant only); therefore, the reverse rate constant is merely $k_{-}^{0}$. Note that this $Y$ number of adhesion molecules belongs to one cell from each bioink, and it is different from $N$ in Eq. (1) that represents the number of involved attaching cells in a cross-section of fusing multicellular systems (a zipper). In fact, based on the zipper model (Fig. 6), each of these two-cell attachments forms an excited state in the zipper, and $N$ is the final number of attached cells after the entire fusion procedure.

By comparing Eqs. (6) and (14), which represent the $\frac{d N_{b}}{d t}$ for nonbiased and biased cell attachments, respectively, one can see the term $\exp \left[\frac{\sigma\left|x_{b}-\lambda\right|\left(\alpha-\frac{1}{2}\left|x_{b}-\lambda\right|\right)}{k_{B} T}\right]$ is the difference between these two situations.

The essential contributor to $\exp \left[\frac{\sigma\left|x_{b}-\lambda\right|\left(\alpha-\frac{1}{2}\left|x_{b}-\lambda\right|\right)}{k_{B} T}\right]$ is the distance between molecules on different cells. The importance of the external force is that it facilitates the cells and their adhesion molecules to overcome this distance and begin developing bonds. Moreover, there is always a competition between nonspecific repulsion and specific binding [39]. Therefore, in reality, electrostatic repulsion, for example, due to the negative charges associated with cell surfaces is compensated for by the external force; hence, the initial states of the bond formation occur much faster than those in nonbiased cell attachment. Perhaps, the empirical results of $t_{0}$ obtained from Figs. 3 and 4 stem from this initial phase of fusion. In this case, $t_{0}$ was found to be 5.23 and $1.12 \mathrm{~h}$ for normal and mechanotransduction-based bioprinting, respectively.

Mathematically, the external force would provide closer locations for adhesion molecules from different cells that shortens the deviation bond length $\left|x_{b}-\lambda\right|$. Changing the deviation bond length may either decrease or increase the term $\exp \left[\frac{\sigma\left|x_{b}-\lambda\right|\left(\alpha-\frac{1}{2}\left|x_{b}-\lambda\right|\right)}{k_{B} T}\right]$. Because the $\sigma$ and $k_{B} T$ are both 

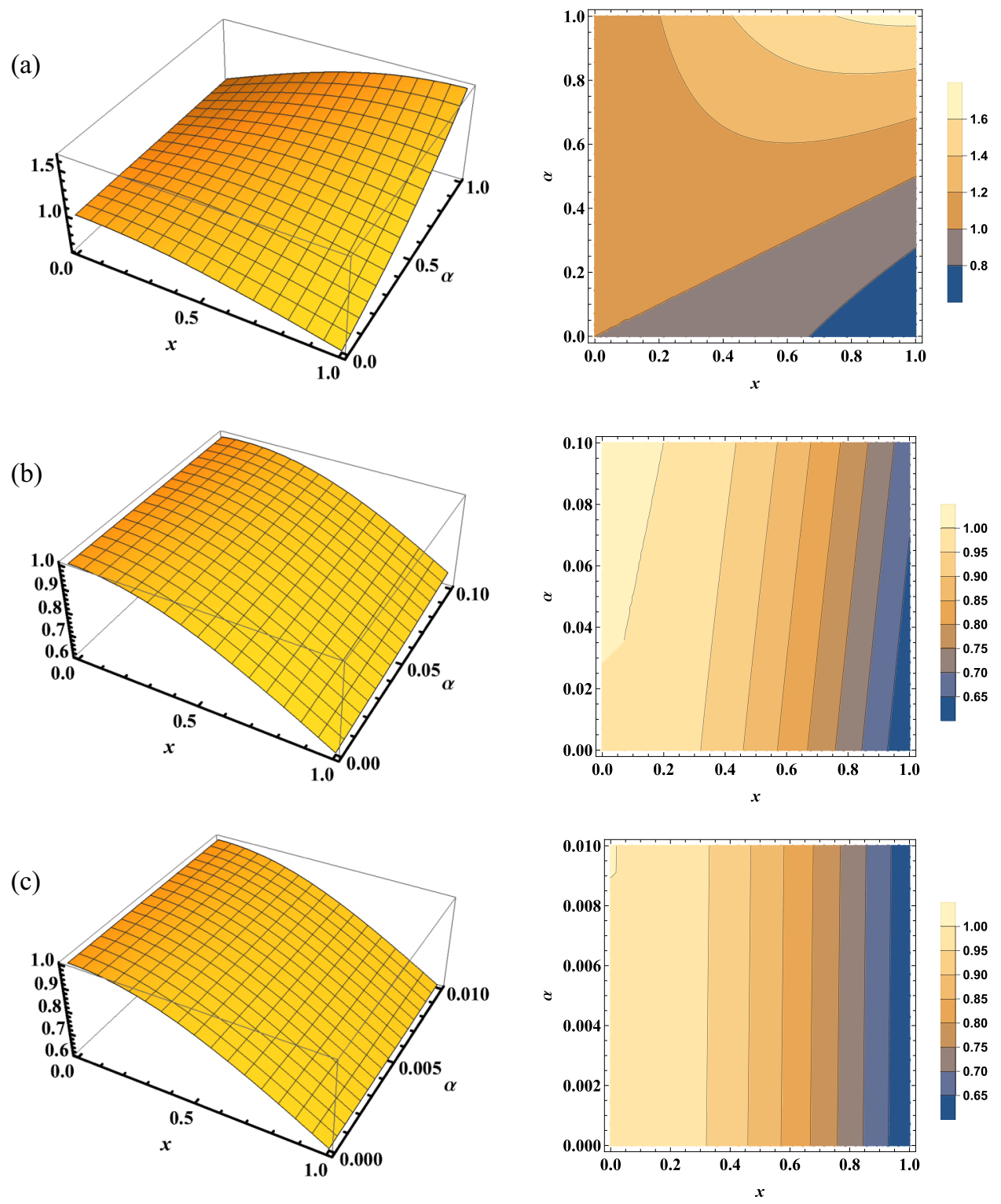

FIG. 7. The effect of deviation bond length on the forward rate constant. (a) $3 \mathrm{D}$ and contour plots for $\exp \left[X\left(\alpha-\frac{1}{2} X\right)\right]$, when $0<\alpha, X<1$. Here, we considered $\left|x_{b}-\lambda\right|=X$. As is shown, $\exp \left[X\left(\alpha-\frac{1}{2} X\right)\right]$ can mathematically be greater than 1 for a range of $\alpha$ and $X$. For example, provided that the $\alpha$ is around 1, the greater values of $x$ also provide values greater than 1 for the exponential term. However, the real ratio for $\alpha$ to $x$ is 0.1 and 0.01 , which are plotted in panels (b) and (c), respectively. It is clear that the smaller deviation bond length would produce greater values for the exponential term in Eq. (14). This confirms the effect of external forces on accelerating the cell attachment process.

constants, one can plot the $3 \mathrm{D}$ graph of $\exp \left[X\left(\alpha-\frac{1}{2} X\right)\right]$ to investigate the effect of deviation bond length. Figure 7(a) depicts the 3D and contour graphs for the $\exp \left[X\left(\alpha-\frac{1}{2} X\right)\right]$, by normalizing the $\alpha$ and $\left|x_{b}-\lambda\right|$ for $0<\alpha,\left|x_{b}-\lambda\right|<1$. It is clear from these plots that small values of $\alpha$ and of $\left|x_{b}-\lambda\right|$ can produce an $\exp \left[\frac{\sigma\left|x_{b}-\lambda\right|\left(\alpha-\frac{1}{2}\left|x_{b}-\lambda\right|\right)}{k_{B} T}\right]$ greater than 1 , as can the greater values of $\left|x_{b}-\lambda\right|$ with those larger $\alpha s$. This indicates that even large deviation bond lengths may produce faster fusion with no need for external forces. However, it is important to note that these mathematical situations are impossible in reality. In fact, it has been shown that, in biological environments, while $\left|x_{b}-\lambda\right|$ are in the scale of a few nanometers $(\mathrm{nm}), \alpha$ s are in the range of 0.1 and $0.01 \mathrm{~nm}$ $[37,38,40]$. Figures $7(b)$ and $7(\mathrm{c})$ show the $3 \mathrm{D}$ and contour graphs for $0<\left|x_{b}-\lambda\right|<1$, with $0<\alpha<0.1$ and $0<\alpha<$ 0.01 , respectively, which clearly shows that smaller values of $\left|x_{b}-\lambda\right|$ can produce larger values for $\exp \left[X\left(\alpha-\frac{1}{2} X\right)\right]$ and consequently produce a greater $\frac{d N_{b}}{d t}$ for biased cell attachment. Therefore, the sooner $N_{b}$ is reached, the more rapidly the two cells are attached.

It is worth mentioning that this method, which is based on the Bell model for specific cell-cell adhesion, aims to determine the rate of bond formation between two single cells. However, the method can also be generalized for a multicellular system and bioink fusion. In this case, the entire procedure of zipper closure is faster when each link of the zipper (two-cell attachment) can be closed faster. Hence, by considering several $[N$ number taken from Eq. (2)] two-cell 


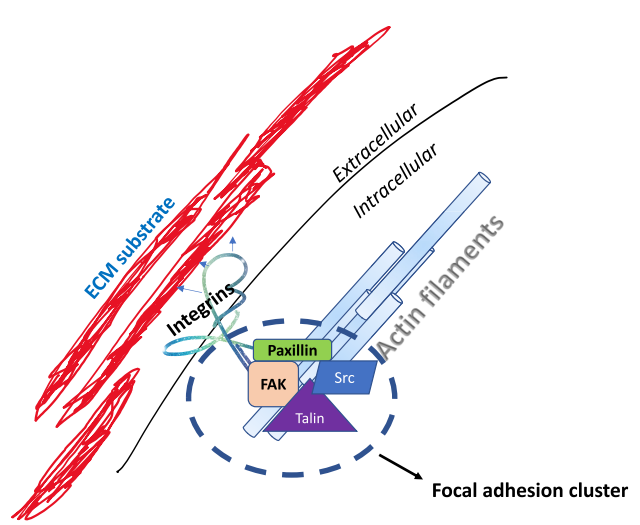

FIG. 8. Schematic representation of some of the key mechanosensing molecules involved in the cell-extracellular matrix (ECM) interaction at the site of focal adhesion. Extracellular changes in stiffness, tension, or other mechanical stimuli are perceived by integrins, which undergo conformational changes and recruit focal adhesion kinase (FAK) to their vicinity on the intracellular side of the membrane. Other proteins aggregate with FAK to form the focal adhesion point (only a few of which are shown in this diagram); the aggregate signaling of these proteins influence the cell's actin cytoskeleton and vice versa.

attachments, we can generalize this method for the fusion of two cylindrical bioinks described using the zipper model. To that end, each excited state defined in the zipper model is the attachment of two cells that is faster in the case of biased attachment. Faster cell attachments result in a quicker fusion in general, thereby accelerating the maturation procedure.

The forward reaction rate in post-bioprinting self-assembly is a more important factor for mechanotransduction-based bioprinting. This causes a faster mass cell migration towards the completion of the fusion process, demonstrating the impact of the external force and mechanotransduction on the acceleration of tissue maturation. The acceleration of organ fabrication is crucial to bringing autologous tissue engineering closer to reality. Therefore, we can envision that different designs and various exerted external forces may enhance the maturation rate of bioprinted tissues. The minimum force required to accelerate tissue formation is that which is just sufficient to overcome repulsive forces, whereas the maximum force is the threshold beyond which cells are damaged. Figure 7 shows an appropriate estimation with which to start optimizing the minimum required force to produce a constant forward rate for biased situations greater than that for unbiased situations. The maximum force must be obtained based on the threshold of resilience of each cell in response to an external force. Therefore, the force must be optimized with respect to two important factors: (1) accelerating the procedure and (2) preventing damage to the cells.

Many of the molecular mechanisms involved in mechanotransduction have been well-described to date, and generally revolve around membrane molecules that elicit cell-cell and cell-microenvironment adhesion. Among the well-studied membrane molecules, integrins, various G-protein and kinase receptors, and ion channels are known to heavily regulate these processes. A key aspect of cell adhesion is the "focal adhesion point" that comprises a plethora of highly concentrated proteins including focal adhesion kinase, paxillin, and others that serve to both anchor the cell to its environment and elicit motility (i.e., the action of a cell's leading edge protruding forward, thereby laying down new focal adhesion points while dissolving the same in the trailing edge on the opposite side). The membrane proteins also serve as nodes that connect to the cell's cytoskeletal components (such as actin filaments). Figure 8 shows a basic schematic of the cell-microenvironment interaction process.

There are few models addressing dynamics of multicellular systems in the developing embryo and tissue engineering [41-45]. Most models have been simulations lacking experimental trials, and were unable to predict the dynamics of multicellular systems that exceeded a certain number of cells. Only the use of cellular particle dynamics was able to predict (but not control) multicellular system behavior [17-19]. Zipper CAMs is designed to investigate the most critical parameters involved in tissue formation and to both predict and control their behavior.

\section{CONCLUSION}

We developed a model for bioprinting tissue formation and maturation that uses statistical mechanics and incorporates the kinetics of cell adhesion molecules. We further devised an alternative bioprinting technology that we used to validate theoretical and mathematical predictions; these were subsequently confirmed in experimental results. As such, we were able to use physics to develop a mathematical language with which to understand and control tissue dynamics experimentally. Our model predicted an acceleration in the maturation of bioprinted tissue using external forces during cellular self-assembly, and ultimately showed a 320\% increase in the fusion rate compared to normal bioprinting. Accelerating tissue maturation is critical for patients who require tissue or organ transplantations immediately. Moreover, despite the tremendous advances in in vitro bioprinting, its usage for clinical applications, including in operating rooms, is so far unrealized. Therefore, a better understanding of the underlying science of cellular self-assembly in bioprinted tissue is essential for accelerating the engineering of tissues and organs for patients with end-stage organ failure, thereby bringing the promise of bioprinted organs a major step closer to reality.

\section{ACKNOWLEDGMENT}

The authors would like to sincerely thank G. Forgacs for fruitful discussions and his dedication to the project.

\section{APPENDIX: ERROR ANALYSIS}

We minimized the residual sum of squares (RSS) to obtain the fits, and obtained RSS values of 0.0098639 for normal bioprinting and 0.00139211 for mechanotransduction bioprinting.

The average characteristic fusion time for six samples printed using normal bioprinting was $9.76731 \mathrm{~h}$ with a standard deviation of $1.027 \mathrm{~h}$. 


\begin{tabular}{ll}
\hline \hline Time point $(t / \tau)$ & Standard deviation for $\sin \theta$ \\
\hline 0.61 & 0.0477 \\
1.02 & 0.038 \\
1.20 & 0.013 \\
2.25 & 0.0211 \\
2.45 & 0.036 \\
3.68 & 0.018 \\
4.91 & 0.007 \\
\hline \hline
\end{tabular}

The average characteristic fusion time for five samples printed using mechanotransduction technology was 3.047 $(\sim 3.05) \mathrm{h}$ with a standard deviation of $0.651 \mathrm{~h}$.

\begin{tabular}{lc}
\hline \hline Time point $(t / \tau)$ & Standard deviation for $\sin \theta$ \\
\hline 0.65 & 0.067 \\
1.31 & 0.118 \\
2.29 & 0.032 \\
3.60 & 0.005 \\
5.24 & 0.005 \\
7.21 & 0.007 \\
9.51 & 0.0004 \\
\hline \hline
\end{tabular}

[1] O. Aubert, P. P. Reese, B. Audry, Y. Bouatou, M. Raynaud, D. Viglietti, C. Legendre, D. Glotz, J.-P. Empana, X. Jouven, C. Lefaucheur, C. Jacquelinet, and A. Loupy, Disparities in acceptance of deceased donor kidneys between the United States and France and estimated effects of increased us acceptance, JAMA Intern. Med. 179, 1365 (2019).

[2] Y. Y. Chan, M. I. Bury, E. M. Yura, M. D. Hofer, E. Y. Cheng, and A. K. Sharma, The current state of tissue engineering in the management of hypospadias, Nat. Rev. Urol. 17, 162 (2020).

[3] W. G. Chang and L. E. Niklason, A short discourse on vascular tissue engineering, npj Regen. Med. 2, 7 (2017).

[4] S. Giwa, J. K. Lewis, L. Alvarez, R. Langer, A. E. Roth, G. M. Church, J. F. Markmann, D. H. Sachs, A. Chandraker, J. A. Wertheim, M. Rothblatt, E. S. Boyden, E. Eidbo, W. P. A. Lee, B. Pomahac, G. Brandacher, D. M. Weinstock, G. Elliott, D. Nelson, J. P. Acker, K. Uygun, B. Schmalz, B. P. Weegman, A. Tocchio, G. M. Fahy, K. B. Storey, B. Rubinsky, J. Bischof, J. A. W. Elliott, T. K. Woodruff, G. J. Morris, U. Demirci, K. G. M. Brockbank, E. J. Woods, R. N. Ben, J. G. Baust, D. Gao, B. Fuller, Y. Rabin, D. C. Kravitz, M. J. Taylor, and M. Toner, The promise of organ and tissue preservation to transform medicine, Nat. Biotechnol. 35, 530 (2017).

[5] R. S. Magalhaes, J. K. Williams, K. W. Yoo, J. J. Yoo, and A. Atala, A tissue-engineered uterus supports live births in rabbits, Nat. Biotechnol. 38, 1280 (2020).

[6] A. M. Raya-Rivera, D. Esquiliano, R. Fierro-Pastrana, E. López-Bayghen, P. Valencia, R. Ordorica-Flores, S. Soker, J. J. Yoo, and A. Atala, Tissue-engineered autologous vaginal organs in patients: A pilot cohort study, Lancet 384, 329 (2014).

[7] A. Raya-Rivera, D. R. Esquiliano, J. J. Yoo, E. López-Bayghen, S. Soker, and A. Atala, Tissue-engineered autologous urethras for patients who need reconstruction: an observational study, Lancet 377, 1175 (2011).

[8] E. Tasciotti, F. J. Cabrera, M. Evangelopoulos, J. O. Martinez, U. R. Thekkedath, M. Kloc, R. M. Ghobrial, X. C. Li, A. Grattoni, and M. Ferrari, The emerging role of nanotechnology in cell and organ transplantation, Transplantation 100, 1629 (2016).

[9] A. Shafiee, E. Ghadiri, J. Kassis, and A. Atala, Nanosensors for therapeutic drug monitoring: implications for transplantation, Nanomedicine (Lond) 14, 2735 (2019).
[10] R. Edri, I. Gal, N. Noor, T. Harel, S. Fleischer, N. Adadi, O. Green, D. Shabat, L. Heller, A. Shapira, I. Gat-Viks, D. Peer, and T. Dvir, Personalized hydrogels for engineering diverse fully autologous tissue implants, Adv. Mater. 31, 1803895 (2018).

[11] A. Shafiee, E. Ghadiri, H. Ramesh, C. Kengla, J. Kassis, P. Calvert, D. Williams, A. Khademhosseini, R. Narayan, G. Forgacs, and A. Atala, Physics of bioprinting, Appl. Phys. Rev. 6, 021315 (2019).

[12] L. Moroni, T. Boland, J. A. Burdick, C. De Maria, B. Derby, G. Forgacs, J. Groll, Q. Li, J. Malda, V. A. Mironov, C. Mota, M. Nakamura, W. Shu, S. Takeuchi, T. B. F. Woodfield, T. Xu, J. J. Yoo, and G. Vozzi, Biofabrication: a guide to technology and terminology, Trends Biotechnol. 36, 384 (2018).

[13] J. Groll, J. A. Burdick, D.-W. Cho, B. Derby, M. Gelinsky, S. C. Heilshorn, T. Jüngst, J. Malda, V. A. Mironov, K. Nakayama, A. Ovsianikov, W. Sun, S. Takeuchi, J. J. Yoo, and T. B. F. Woodfield, A definition of bioinks and their distinction from biomaterial inks, Biofabrication 11, 013001 (2018).

[14] C. Norotte, F. S. Marga, L. E. Niklason, and G. Forgacs, Scaffold-free vascular tissue engineering using bioprinting, Biomaterials 30, 5910 (2009).

[15] C. Owens, F. Marga, and G. Forgacs, Bioprinting of Nerve, edited by A. Atala and J. J. Yoo, in Essentials of 3D Biofabrication and Translation (Elsevier, San Diego, 2015), pp. 379-394.

[16] N. Khoshnood and A. Zamanian, A comprehensive review on scaffold-free bioinks for bioprinting, Bioprinting 19, e00088 (2020).

[17] M. McCune, A. Shafiee, G. Forgacs, and I. Kosztin, Predictive modeling of post bioprinting structure formation, Soft Matter 10, 1790 (2014).

[18] I. Kosztin, G. Vunjak-Novakovic, and G. Forgacs, Colloquium: Modeling the dynamics of multicellular systems: Application to tissue engineering, Rev. Mod. Phys. 84, 1791 (2012).

[19] A. Shafiee, M. McCune, G. Forgacs, and I. Kosztin, Post-deposition bioink self-assembly: A quantitative study, Biofabrication 7, 045005 (2015).

[20] K. Zhang, S. Wang, C. Zhou, L. Cheng, X. Gao, X. Xie, J. Sun, H. Wang, M. D. Weir, M. A. Reynolds, N. Zhang, Y. Bai, and $\mathrm{H}$. H. K. Xu, Advanced smart biomaterials and constructs for hard tissue engineering and regeneration, Bone Res. 6, 31 (2018). 
[21] F. Martino, A. R. Perestrelo, V. Vinarský, S. Pagliari, and G. Forte, Cellular mechanotransduction: From tension to function, Front Physiol. 9, 507 (2018).

[22] T. Mammoto, A. Mammoto, and D. E. Ingber, Mechanobiology and developmental control, Annu. Rev. Cell Dev. Biol. 29, 27 (2013).

[23] B. Hashmi, T. Mammoto, J. Weaver, T. Ferrante, A. Jiang, E. Jiang, J. Feliz, and D. E. Ingber, Mechanical induction of dentin-like differentiation by adult mouse bone marrow stromal cells using compressive scaffolds, Stem Cell Res. 24, 55 (2017).

[24] S. Chatterjee, Endothelial mechanotransduction, redox signaling and the regulation of vascular inflammatory pathways, Front Physiol. 9, 314 (2018).

[25] Z. Sun, S. S. Guo, and R. Fässler, Integrin-mediated mechanotransduction, J. Cell Biol. 215, 445 (2016).

[26] J. J. Saucerman, P. M. Tan, K. S. Buchholz, A. D. McCulloch, and J. H. Omens, Mechanical regulation of gene expression in cardiac myocytes and fibroblasts, Nat. Rev. Cardiol. 16, 361 (2019).

[27] L. R. Smith, S. Cho, and D. E. Discher, Stem cell differentiation is regulated by extracellular matrix mechanics, Physiology 33, 16 (2018).

[28] G. Forgacs, R. A. Foty, Y. Shafrir, and M. S. Steinberg, Viscoelastic properties of living embryonic tissues: a quantitative study, Biophys J. 74, 2227 (1998).

[29] R. A. Foty and M. S. Steinberg, Differential adhesion in model systems, Wiley Interdiscip. Rev. Dev. Biol. 2, 631 (2013).

[30] S. Ji and M. Guvendiren, Recent advances in bioink design for 3D bioprinting of tissues and organs, Front. Bioeng. Biotechnol. 5, 014102 (2017).

[31] G. Bell, Models for the specific adhesion of cells to cells, Science 200, 618 (1978).

[32] A. Shafiee, E. Ghadiri, D. Williams, and A. Atala, Physics of cellular self-assembly: A microscopic model and mathematical framework for faster maturation of bioprinted tissues, Bioprinting 14, e00047 (2019).

[33] A. Shafiee, C. Norotte, and E. Ghadiri, Cellular bioink surface tension: A tunable biophysical parameter for faster maturation of bioprinted tissue, Bioprinting 8, 13 (2017).

[34] N. L'Heureux, T. N. McAllister, and L. M. de la Fuente, Tissueengineered blood vessel for adult arterial revascularization, $\mathrm{N}$. Engl. J. Med. 357, 1451 (2007).

[35] N. L'Heureux, N. Dusserre, G. Konig, B. Victor, P. Keire, T. N. Wight, N. A. F. Chronos, A. E. Kyles, C. R. Gregory, G. Hoyt, R. C. Robbins, and T. N. McAllister, Human tissue-engineered blood vessels for adult arterial revascularization, Nat. Med. 12, 361 (2006).

[36] Foty and Steinberg, The differential adhesion hypothesis: a direct evaluation, Dev. Biol. 278, 255 (2005).

[37] S. K. Bhatia, M. R. King, and D. A. Hammer, The state diagram for cell adhesion mediated by two receptors, Biophys. J. 84, 2671 (2003).

[38] E. Lomakina and R. E. Waugh, inPrinciples of Cellular Engineering: Understanding the Biomedical Interface, edited by M. King (Elsevier, San Diego, 2006), pp. 105-122.

[39] G. Bell, M. Dembo, and P. Bongrand, Cell adhesion: Competition between nonspecific repulsion and specific bonding, Biophys. J. 45, 1051 (1984).

[40] M. J. Smith, E. L. Berg, and M. B. Lawrence, A direct comparison of selectin-mediated transient, adhesive events using high temporal resolution, Biophys. J. 77, 3371 (1999).

[41] G. M. Odell, G. Oster, P. Alberch, and B. Burnside, The mechanical basis of morphogenesis I. Epithelial folding and invagination, Dev. Biol. 85, 446 (1981).

[42] F. Graner and J. A. Glazier, Simulation of Biological Cell Sorting Using a Two-Dimensional Extended Potts Model, Phys. Rev. Lett. 69, 2013 (1992).

[43] E. Palsson and H. G. Othmer, A model for individual and collective cell movement in dictyostelium discoideum, Proc. Natl. Acad. Sci. USA 97, 10448 (2000).

[44] D. Drasdo and G. Forgacs, Modeling the interplay of generic and genetic mechanisms in cleavage, blastulation, and gastrulation, Dev. Dyn. 219, 182 (2000).

[45] T. J. Newman, Modeling multicellular systems using subcellular elements, Math. Biosci. Eng. 2, 613 (2005). 